\title{
MagnEdit-interacting factors that recruit DNA-editing enzymes to single base targets
}

Jennifer L McCann ${ }^{1,2,3,4,5}$, Daniel J Salamango ${ }^{1,2,3,4}$, Emily K Law ${ }^{1,2,3,4,5}$, William L Brown ${ }^{1,2,3,4}$ (D), Reuben S Harris ${ }^{1,2,3,4,5}$ (D)

\begin{abstract}
Although CRISPR/Cas9 technology has created a renaissance in genome engineering, particularly for gene knockout generation, methods to introduce precise single base changes are also highly desirable. The covalent fusion of a DNA-editing enzyme such as APOBEC to a Cas9 nickase complex has heightened hopes for such precision genome engineering. However, current cytosine base editors are prone to undesirable off-target mutations, including, most frequently, target-adjacent mutations. Here, we report a method to "attract" the DNA deaminase, APOBEC3B, to a target cytosine base for specific editing with minimal damage to adjacent cytosine bases. The key to this system is fusing an APOBECinteracting protein (not APOBEC itself) to Cas9n, which attracts nuclear APOBEC3B transiently to the target site for editing. Several APOBEC3B interactors were tested and one, hnRNPUL1, demonstrated proof-of-concept with successful C-to-T editing of episomal and chromosomal substrates and lower frequencies of target-adjacent events.
\end{abstract}

DoI 10.26508/lsa.201900606 | Received 17 November 2019 | Revised 14 February 2020 | Accepted 17 February 2020 | Published online 24 February 2020

\section{Introduction}

The original $\mathrm{BE} 3$ cytosine base editor (CBE) comprised the rat APOBEC1 deaminase fused to the N-terminal end of a Cas9 nickase (Cas9n D10A (1)). Appropriate gRNAs are able to target this assembly to genomic cytosine bases and facilitate high-frequency editing (10-90\% depending on a number of variables including distance between target cytosine and protospacer adjacent motif) $(1,2)$. However, this technology is prone to a number of off-target effects, including RNA editing $(3,4)$, random genomic DNA editing $(5,6,7,8)$, and most frequently target-adjacent editing $(1,2,5,9,10)$. The latter problem is due predominantly, if not exclusively, to deamination of single-stranded DNA cytosines located adjacent to the desired target cytosine in the same gRNA-displaced R-loop. This issue has been diminished-but not eliminated-by mutating APOBEC1 ( 3,4 , $10,11)$, trying different DNA deaminase family members $(12,13,14,15$,
16, 17, 18), mutating Cas9 $(10,19,20,21,22,23,24)$, and leveraging different Cas enzymes $(11,16,24,25)$. However, an invariant feature of almost all current designs is covalent fusion of the deaminase to the Cas9 complex, which traps the tethered deaminase locally and inextricably links both on-target (desirable) and target-adjacent (undesirable) cytosine deamination events (schematic in Fig 1A).

We hypothesize that non-covalent methods to "attract" a DNA cytosine deaminase to a particular genomic cytosine target will decouple the fates of on-target and target-adjacent editing events and thereby enhance the likelihood of achieving precise single base substitution mutations. A key to implementing this noncovalent strategy is identifying appropriate APOBEC-interacting proteins, which bind the deaminase without blocking the active site from engaging a target cytosine. Such interacting proteins can then be tethered to a Cas9n/gRNA complex and used to "attract" a co-expressed APOBEC enzyme (exogenous or endogenous) to edit a particular genomic target cytosine. Inspired by the analogy to magnetism, this system is called MagnEdit (schematic in Fig 1B).

\section{Results}

Covalent CBE versus non-covalent MagnEdit technology for DNA cytosine base editing

As an initial test of MagnEdit, we fused APOBEC3B (A3B)-interacting proteins from the literature (simian immunodeficiency virus [SIV] Vif (26), hnRNPK (27)) and proteomic screens (CDK4 (28) and McCann et al, unpublished) to the $\mathrm{N}$-terminal end of Cas9n and asked whether these complexes are able to recruit $A 3 B$ to edit an episomal eGFP reporter (13) in 293T cells (TC to TT schematic in Fig 1B and actual eGFP gRNA target sequence in Fig $1 \mathrm{C}$ inset). Because of simultaneous overexpression of reaction components following cotransfection, including A3B, a low level of eGFP-positive cells $(\sim 1-2 \%)$ was observed in the absence of a gRNA and a candidate interacting protein (reactions represented by gray and black bars in Fig 1C). Interestingly, addition of eGFP Leu202-targeting gRNA (again without an interactor) enabled higher levels of eGFP editing by A3B

${ }^{1}$ Department of Biochemistry, Molecular Biology and Biophysics, University of Minnesota, Minneapolis, MN, USA ${ }^{2}$ Institute for Molecular Virology, University of Minnesota, Minneapolis, MN, USA ${ }^{3}$ Masonic Cancer Center, University of Minnesota, Minneapolis, MN, USA ${ }^{4}$ Center for Genome Engineering, University of Minnesota, Minneapolis, MN, USA ${ }^{5}$ Howard Hughes Medical Institute, University of Minnesota, Minneapolis, MN, USA

Correspondence: rsh@umn.edu 
A

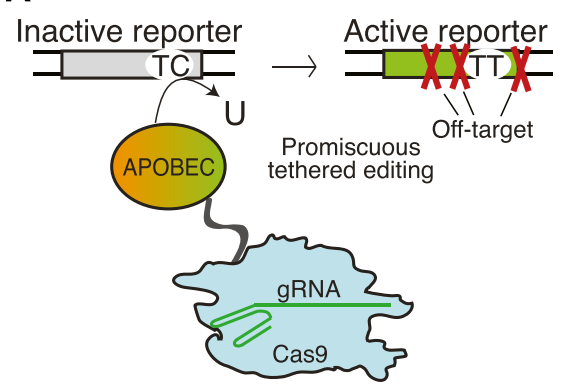

B

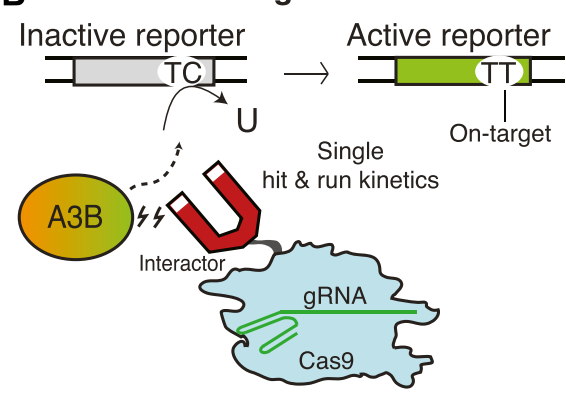

C

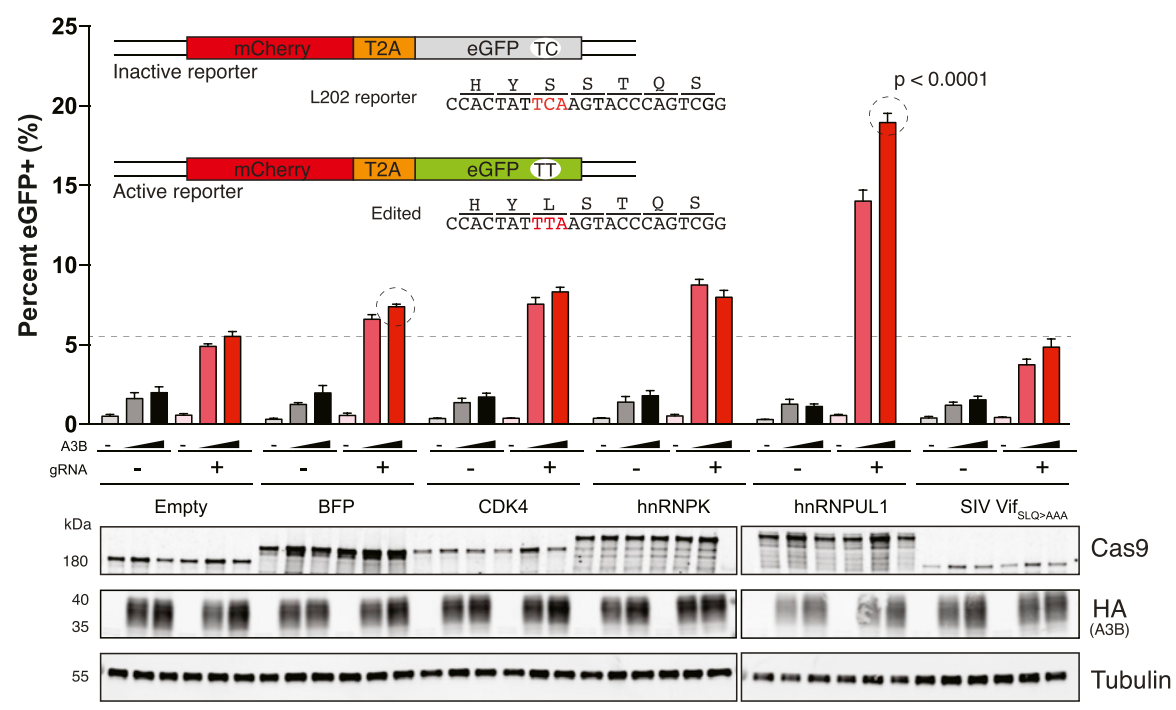

Figure 1. Covalent CBE versus non-covalent MagnEdit technology for DNA cytosine base editing.

(A) Schematic of current CBE methodology with APOBEC-Cas9n/gRNA editosome engaging the eGFP Leu202 reporter. Target-adjacent mutations are indicated by red X's. (B) Schematic of MagnEdit with interactor-Cas9n/gRNA complex recruiting untethered A3B to the eGFP Leu202 reporter. (C) Quantification of episomal eGFP reporter editing activity of the indicated MagnEdit complexes in 293T cells ( $n=3$ biologically independent experiments, average \pm SD, $P<0.0001$ by unpaired $t$ test for circled reactions). The immunoblots below are from one of these experiments. The inset schematic shows the eGFP Leu202 reporter, the DNA region matching the gRNA, and the target cytosine in red.
( 5-7\%; empty-Cas9n plus gRNA reaction in Fig 1C). Unfortunately, most MagnEdit complexes failed to stimulate editing beyond these background levels or those caused by a non-interacting blue fluorescent protein (BFP)-Cas9n control (Fig 1C). SIV Vif (SLQ-AAA)-Cas9n even yielded lower overall frequencies of background editing, likely because of poorer expression relative to other MagnEdit constructs (the SLQ-AAA was necessary to prevent Vif from binding ELOC and triggering A3B degradation (26)). However, one MagnEdit construct, hnRNPUL1-Cas9n, was clearly capable of recruiting A3B in a dosedependent manner to catalyze editing and activation of the eGFP reporter (Fig 1C). Editing frequencies due to hnRNPUL1-Cas9n were at least twofold higher than the BFP-Cas9n/gRNA-induced background in these transient transfection experiments $(P<0.0001$ by unpaired $t$ test).

\section{Chromosomal DNA editing by MagnEdit}

Next, we analyzed chromosomal DNA editing by MagnEdit. The same eGFP Leu202 reporter was integrated into the genome of 293T cells by low MOI lentiviral transduction followed by hygromycin selection to ensure that every cell has one editing target (uniform mCherrypositive population confirmed by flow cytometry). This pool was then transfected, as above, with the panel of A3B interactor-Cas9n complexes with or without the Leu202-targeting gRNA in the presence or absence of exogenous A3B. Also, as above, empty-Cas9n and BFP-
Cas9n were used as negative controls, and most MagnEdit complexes showed no activity above background levels. Flow cytometry noise was the likely source of these low background levels of eGFP positivity because no difference was seen here with/without the eGFP Leu202targeting gRNA or different amounts of A3B. However, in agreement with episomal editing data, hnRNPUL1 MagnEdit complexes yielded a dose-dependent increase in $\mathrm{A} 3 \mathrm{~B}$ editing (quantification and representative immunoblots in Fig $2 \mathrm{~A} ; P<0.0009$ by unpaired $t$ test). As expected, all components of the MagnEdit reaction were required for chromosomal DNA editing (hnRNPUL1-Cas9n complex, Leu202 gRNA, and A3B-HA; Fig 2B).

To further investigate the mechanistic requirements for MagnEdit, we asked whether the nuclear import activity of A3B is required. A3B is the only constitutively nuclear human APOBEC family member $(29,30,31)$ and nuclear localization is predicted to be essential for MagnEdit activity. Recent studies have combined to delineate a non-canonical nuclear import mechanism involving multiple A3B surface residues in two distinct patches (31). Indeed, two previously characterized import-defective mutants of A3B, V54D (29), and chimera 22-32 (31) were no longer capable of editing the chromosomal eGFP Leu202 reporter (Fig 2C). These amino acid substitutions localize to the $\mathrm{N}$-terminal regulatory domain of $\mathrm{A} 3 \mathrm{~B}$ and the editing phenotype is indistinguishable from that of a C-terminal domain catalytic mutant (CM in Fig 2C). In addition, the 
A

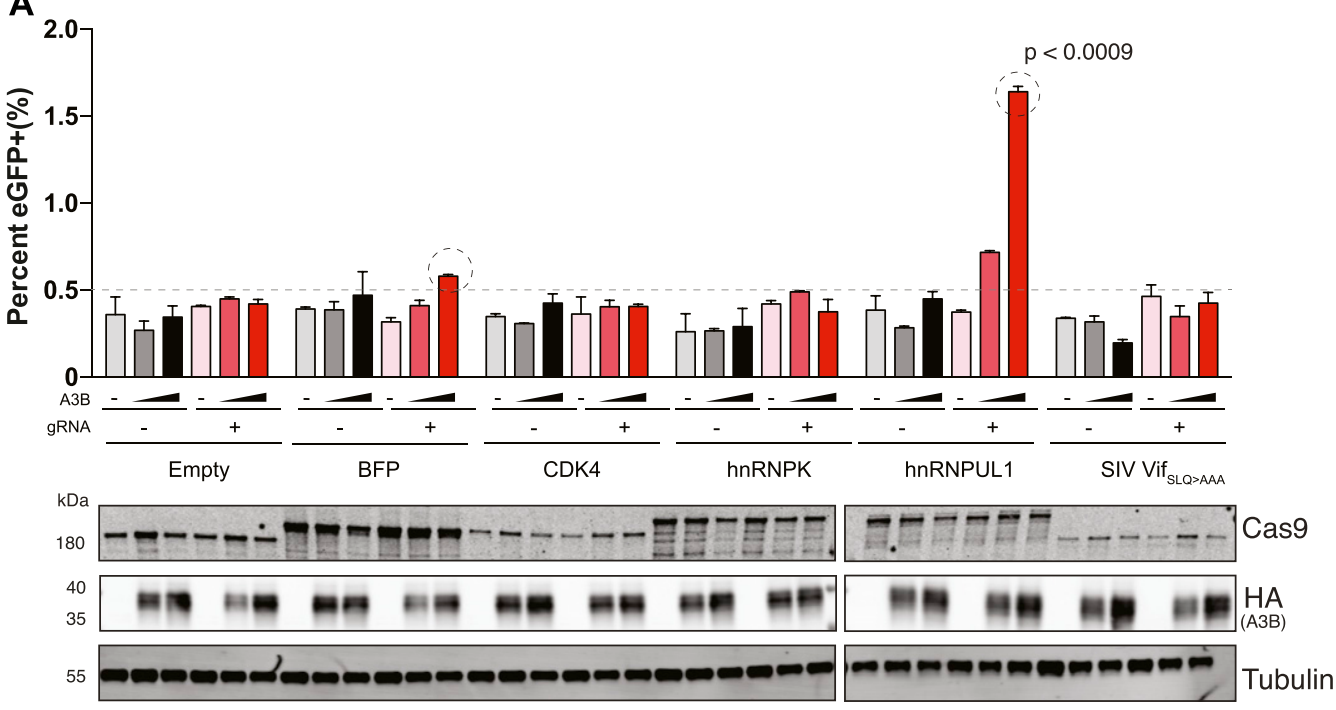

B 1.5

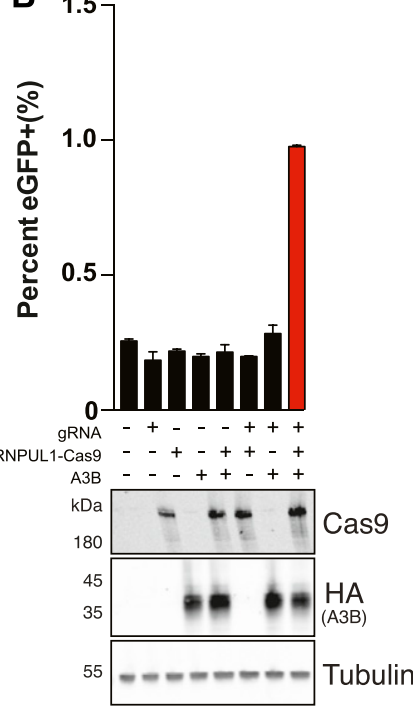

C

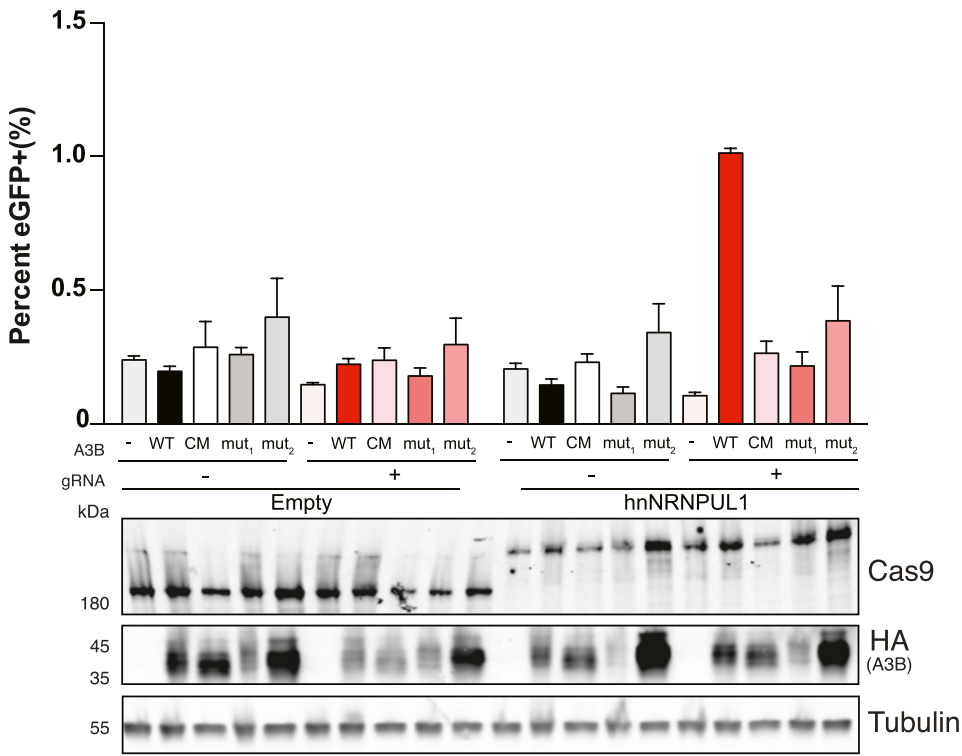

D 2.0

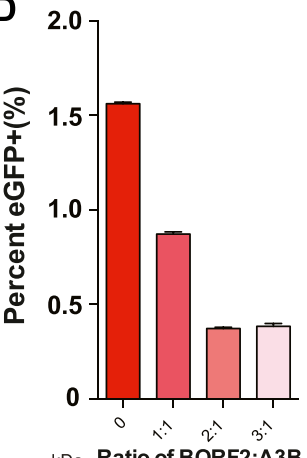

RDatio of BORF2:A3B

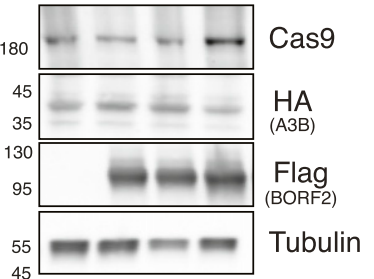

Figure 2. Chromosomal DNA editing by MagnEdit.

(A) Quantification of chromosomal eGFP reporter editing activity of the indicated MagnEdit complexes in 293T cells $(n=3$ biologically independent experiments, average \pm SD, $P<$ 0.0009 by unpaired $t$ test for circled reactions). The immunoblots below are from one of these experiments. (B, C, D) Chromosomal eGFP editing activity for reactions containing the indicated components $(n=3$, average $\pm S D)$. The immunoblots below each histogram are from one of the experiments.

chromosomal DNA-editing reaction can be suppressed in a dosedependent manner by BORF2, a recently discovered A3B antagonist encoded by Epstein-Barr virus (32) (Fig 2D).

\section{L202 reporter editing by CBE versus MagnEdit}

DNA sequencing was used to compare the ratios of on-target and targetadjacent editing by a current CBE (A3B-Cas9n) (13) and the MagnEdit complex described here (A3B plus hnRNPUL1-Cas9n). A3B-Cas9n was used for these comparisons because its catalytic domain is less promiscuous than BE3 (13) and it provides an isogenic comparison for covalent versus non-covalent editing reactions catalyzed by A3B. As above, chromosomal DNA editing was performed by transfecting Cherry- positive 293T pools with the eGFP Leu202 gRNA expression vector and plasmids encoding either A3B-Cas9n or hnRNPUL1-Cas9n with a separate vector for A3B. FACS was used $72 \mathrm{~h}$ post-transfection to isolate eGFP-positive pools for target recovery and deep sequencing. As indicated by bright eGFP-positive signals in each editing reaction $72 \mathrm{~h}$ posttransfection, both editing technologies activated the reporter with the A3B CBE appearing approximately fourfold more efficient (6.1\% for A3BCas9n and 1.5\% for A3B plus hnRNPUL1-Cas9n; Fig 3A). In each instance, FACS resulted in enrichment of similar numbers of eGFP-positive cells for deep sequencing of the Leu202 target codon and flanking DNA (98\% for A3B-Cas9n and 99\% for A3B plus hnRNPUL1-Cas9n; Fig 3A).

As negative controls, parallel reactions without gRNAs were directly converted to genomic DNA for deep sequencing and no 
A

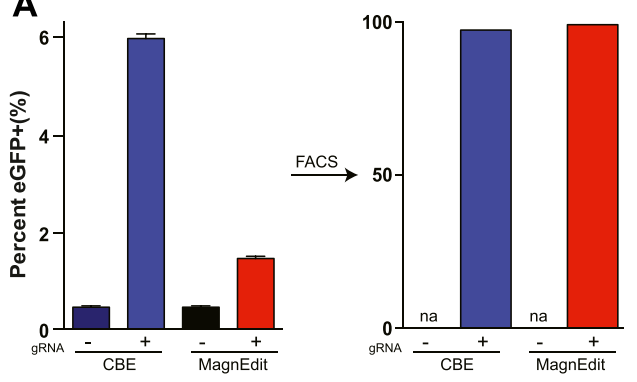

B

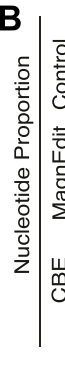

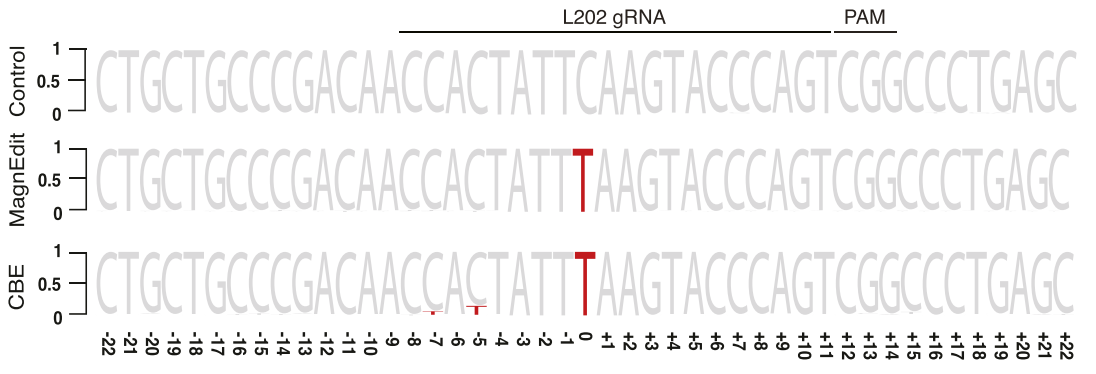

Figure 3. Target-adjacent editing by CBE versus MagnEdit.

(A) Quantification of eGFP-positive 293T cells (eGFP Leu202 edited) post-editing and pre-/post-enrichment by FACS for the indicated editing reactions ( $\mathrm{n}=3$ technical replicate experiments, average \pm SD). (A, B) Sequence logos summarizing MiSeq data from the same reactions as panel (A). The consensus sequence matches the singlestranded DNA region displaced by gRNA annealing with the target cytosine. Red coloring highlights mutations that occurred in $>5 \%$ of the MiSeq reads for each reaction (numbers are nucleobase distances $5^{\prime}$ or $3^{\prime}$ of the target " $C$ ").

target cytosine mutations were observed. As anticipated above and from prior studies (13), the inclusion of a gRNA enabled both technologies to restore functionality to eGFP codon 202 (TCA [Ser] to TTA [Leu]; represented by a red $\mathrm{T}$ and normalized to 1 for comparisons in Fig 3B). However, target-adjacent editing frequencies were clearly different for these two different base editing technologies. The covalently tethered A3B-Cas9n CBE caused high frequencies of target-adjacent editing within the R-loop created by gRNA binding ( $14.8 \%$ at the -5 position and $6.4 \%$ at the -7 position in Fig 3B). In contrast, the hnRNPUL1-Cas9n MagnEdit system showed lower target-adjacent editing within the gRNAbinding region (2.5\% at both -5 and -7 positions in Fig $3 \mathrm{~B}$ ).

\section{Chromosomal DNA editing by CBE versus MagnEdit}

To further investigate the accuracy of the MagnEdit system, we compared ratios of on-target and target-adjacent editing by a current CBE (A3BCas9n) (13) and the MagnEdit complex described here (A3B plus hnRNPUL1-Cas9n) at two genomic loci, FANCF and EMX1, reported previously (1). As above, chromosomal DNA editing was performed by transfecting Cherry-positive 293T pools with gRNAs targeting both the eGFP Leu202 reporter and FANCF or EMX1 and plasmids encoding either A3B-Cas9n or hnRNPUL1-Cas9n with a separate vector for A3B. FACS was used $72 \mathrm{~h}$ post-transfection to isolate eGFP-positive pools for target DNA recovery and deep sequencing. Similar to results in Fig 3A, both editing technologies activated the eGFP reporter with, again, the A3B CBE appearing approximately fourfold more efficient (Fig 4A and E).

As negative controls, parallel reactions without gRNAs were directly converted to genomic DNA for deep sequencing and no target cytosine mutations were observed in FANCF or EMX1 (control reactions in Fig $4 \mathrm{~B}$ and $\mathrm{F}$ ). However, upon inclusion of appropriate gRNAs targeting these genes, clear differences in accuracy were observed between these two different base editing technologies. Similar to prior literature for FANCF editing by BE3 (1), the covalently tethered A3B-Cas9n CBE caused high frequencies of targetadjacent editing within the R-loop created by gRNA binding ( $42 \%$ at the +1 position and $35 \%$ at the +2 position in Fig 4B). It also caused significant off-target editing at the -9 position, which is just upstream of the gRNA-binding region (13.9\% in Fig 4B). In contrast, the hnRNPUL1-Cas9n MagnEdit system showed significantly lower target-adjacent editing within the gRNA-binding region and no detectable editing outside of the gRNA-binding region ( $13 \%$ at the +1 position, $20 \%$ at the +2 position, and $0.5 \%$ at the -9 position in $\mathrm{Fig}$ $4 \mathrm{~B})$. Although target-adjacent editing is higher in FANCF than in the eGFP L202 reporter, this is likely due to the trinucleotide context of FANCF being "TCC" rather than "TCA" (i.e., TCC is a suboptimal context for $\mathrm{A} 3 \mathrm{~B}$ as shown by biochemical and structural studies (33)). Nevertheless, upon consideration of all possible editing permutations within the gRNA-binding region (on-target and target-adjacent events), the hnRNPUL1-Cas9n MagnEdit system shows a twofold increase in on-target editing in comparison to the covalently tethered A3B-Cas9n CBE (19\% versus 9\% in Fig 4C and D, respectively). The hnRNPUL1-Cas9n MagnEdit system yields correspondingly fewer target-adjacent editing events than the $\mathrm{A} 3 \mathrm{~B}$ Cas9n CBE system (21.8\% versus $45.5 \%$ in Fig 4 C and D, respectively).

Similar trends were evident for the chromosomal EMX1 locus. The covalently tethered A3B-Cas9n CBE caused high frequencies of target-adjacent editing within the R-loop created by the gRNA binding ( $58.5 \%$ at the +1 position in Fig $4 \mathrm{~F}$ ). In contrast, the hnRNPUL1Cas9n MagnEdit system showed more than threefold lower targetadjacent editing within the gRNA-binding region (15.0\% at the +1 position in Fig 4F). Again, this genomic target has a trinucleotide context of "TCC" rather than "TCA," so editing results were broken down into trinucleotide contexts for further consideration. The hnRNPUL1-Cas9n MagnEdit system specifically edited the target " $\mathrm{C}$," whereas the covalently tethered A3B-Cas9n CBE was less specific ( $49 \%$ versus $18.2 \%$ on-target editing, respectively, Fig $4 \mathrm{G}$ and $H$ ). These results combine to demonstrate that the MagnEdit system yields higher frequencies of on-target editing with significantly lower frequencies of target-adjacent editing events. In addition, higher FANCF and EMX1 on-target editing frequencies and similar adjacent offtarget trends were evident for MagnEdit versus the covalently tethered A3B-Cas9n CBE in eGFP-negative pools (Fig S1). These additional results from sequencing the "dark" population suggested that on-target chromosomal editing events may far exceed those that yield functional correction of the eGFP Leu202 reporter.

\section{Discussion}

This study describes a fundamentally different approach to single base editing through the use of non-covalent interactions to "attract" a DNA cytosine deaminase to a single target cytosine. A3B is 


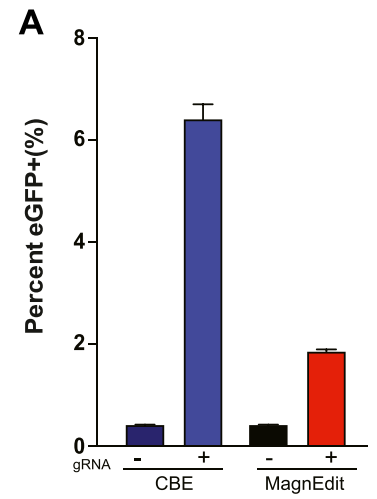

C

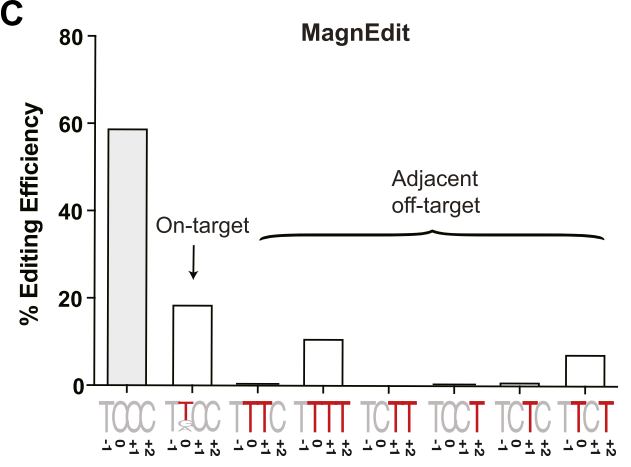

B
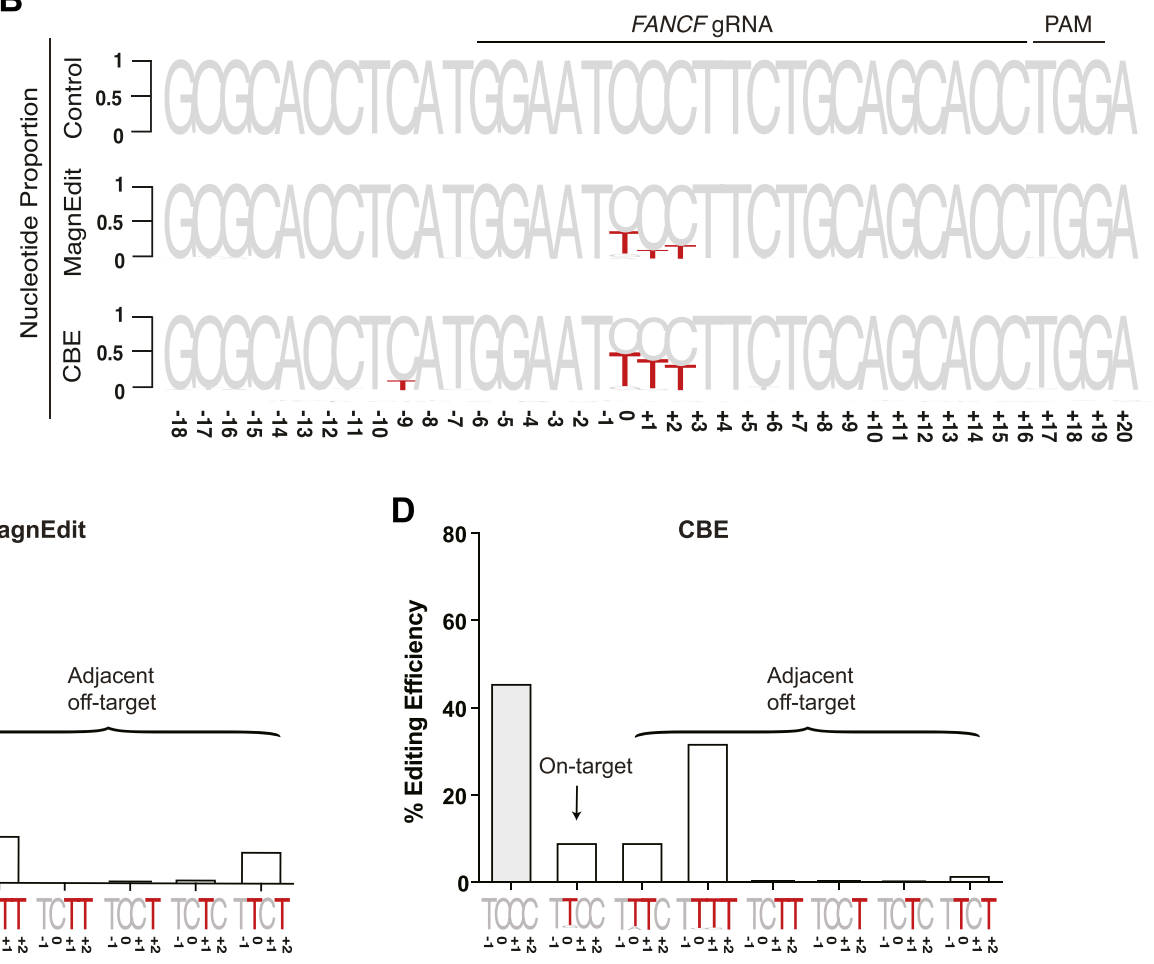

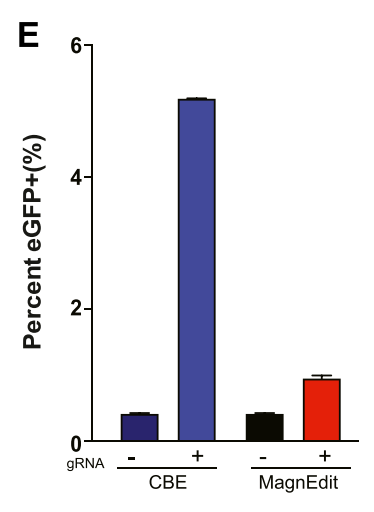

$\mathbf{F}$

G

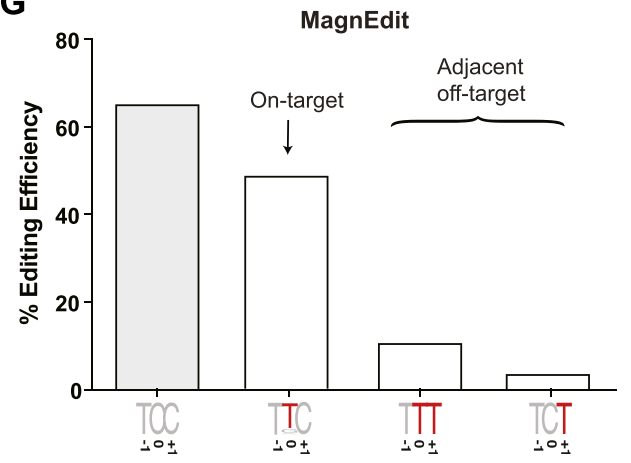

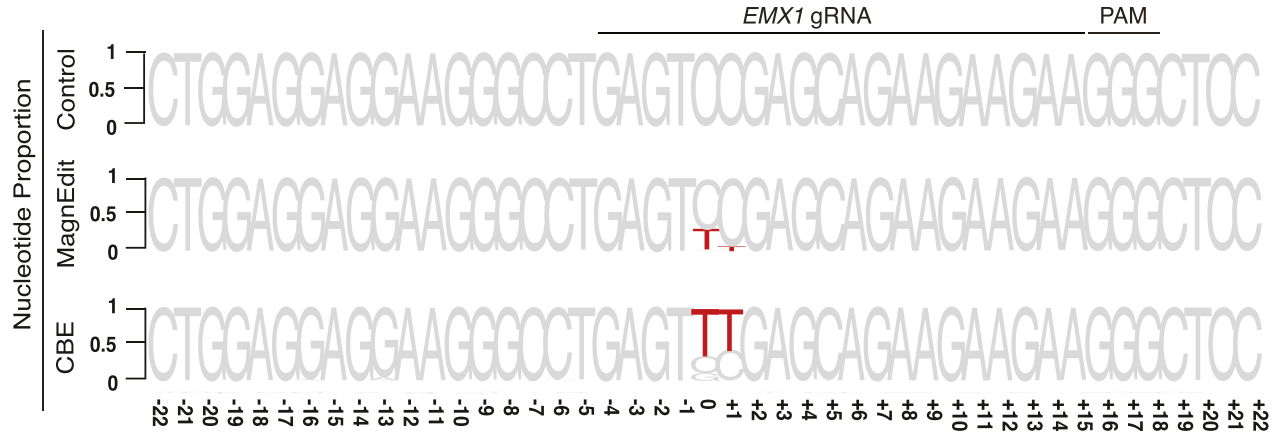

H

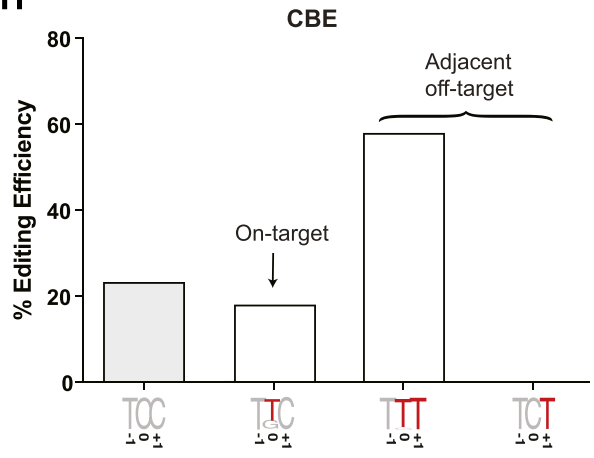

Figure 4. Chromosomal DNA editing by cytosine base editor (CBE) versus MagnEdit.

(A) Quantification of eGFP-positive 293T cells (eGFP Leu202 edited with co-delivery of FANCF gRNA) post-editing and pre-enrichment by FACS for the indicated editing reactions ( $n=3$ technical replicate experiments, average \pm SD). (A, B) Sequence logos summarizing Miseq data of FANCF from the same reactions as panel (A). The consensus sequence matches the single-stranded DNA region displaced by gRNA annealing with the target cytosine. Red coloring highlights base substitution mutations that occurred in $>5 \%$ of the MiSeq reads for each reaction (numbers are nucleobase distances $5^{\prime}$ or $3^{\prime}$ of the target " $C^{\prime \prime}$ ). (B, C) Quantification of single nucleobase substitution mutations from the MagnEdit reaction shown in panel (B). (B, D) Quantification of single nucleobase substitution mutations from the CBE reaction shown in 
desirable for this application because it is normally nuclear (not shuttling or cytoplasmic like related family members) $(29,30,31,34,35)$ and, because of active site structural constraints $(33,36,37)$, unlikely to elicit RNA level off-target editing events as documented recently for $B E 3$ and $A 3 A$ CBEs $(3,4)$. A variety of techniques may be used in the future to identify additional APOBEC-interacting "baits" for the MagnEdit system (proteomic, genetic, and directed-evolution,). Naturally or artificially engineered antibodies may also be effective. Similar MagnEdit approaches may also benefit adenosine base editing systems. Although we have not observed nor found literature indicating potential side effects of overexpressing hnRNPUL1 in cells, heterologous attractants such as single-chain antibodies may be even better for promoting the non-covalent editing of single target cytosine bases.

In general, proteins such as hnRNPUL1 that interact with the noncatalytic $\mathrm{N}$-terminal domain of A3B may be more effective than those that bind the catalytic C-terminal domain simply because they are less likely to interfere with catalytic activity. For instance, EBV BORF2 is the only A3B catalytic domain interactor described till date (32) and, as shown here, it potently blocks editing in the MagnEdit system. However, not all A3Binteracting proteins are likely to be effective in the MagnEdit system because affinities may be too low, nuclear access may not be allowed, and/or binding confirmations may be unproductive (e.g., CDK4, hnRNPK, and SIV Vif). Although others have used non-covalent methods such as SunTagging (38) and MS2 (18) to recruit DNA methyltransferase 3A (DNMT3A) and AID, respectively, these methods rely upon very highaffinity binding for positive results $(39,40)$. It is likely the high on-target and low target-adjacent editing of the MagnEdit system is due to an optimal affinity between hnRNPUL1 and A3B, which leaves A3B untethered and able to generate greater than random "hit-and-run" kinetics at the target "C." Methods such as SunTagging and MS2 may be efficient for editing enzyme recruitment but are unlikely to differ from covalent tethering in terms of increased accuracy due to high on-rates and low offrates. Moreover, with additional optimization, it is likely that the MagnEdit system may be used with endogenous A3B and/or related A3 enzymes and, therefore, mitigate risks associated with overexpressing enzymes exogenously. Overall, the non-covalent MagnEdit system is attractive for helping to minimize off-target effects and ultimately enable true single base editing even though higher fidelities may come at a cost of lower overall efficiencies.

\section{Materials and Methods}

\section{Cell lines}

293T and 293T-Leu202 cells were cultured in RPMI 1640 supplemented with $10 \%$ FBS and penicillin-streptomycin. The chromosomal 293T-Leu202 reporter line was constructed using viral transduction followed by hygromycin selection (detailed below).

\section{Constructs}

The rat APOBEC1-XTEN-CaS9n-UGI-NLS construct (BE3) was provided by David Liu (1). Interactor CDNA sequences were cloned into the BE3 vector in place of APOBEC1 using standard PCR subcloning techniques, thereby creating CMV-driven constructs with gene-of-interest followed by an XTEN linker to Cas9n (D10A)-UGI-NLS. GenBank accession numbers for BFP (MK178577.1), CDK4 (NM_000075.4), hnRNPK (NM_031263.4), and hnRNPUL1 (EU831487.1). SIV Vif was subcloned from a reported construct $(26,41)$. Leu202 gRNA, NS gRNA, empty-Cas9n-UGINLS, and Leu202 reporter (pLenti-CMV-mCherry-T2A-eGFP) have been reported (13). EMX1 and FANCF guide constructs were cloned into LentiCRISPR1000 (42) via Golden Gate cloning using the Esp3I sites. EMX1 and FANCF target sequences have been reported (1). All constructs were confirmed by Sanger DNA sequencing (GeneWiz). pcDNA3.1-3xHA, A3Bi$3 \times \mathrm{XHA}$, and $\mathrm{A}_{3 \mathrm{Bi} 54 \mathrm{D}}-3 \mathrm{xHA}$ have been reported (29), and $\mathrm{A}_{3} \mathrm{~B}_{\text {chim22-32 }}-3 \times \mathrm{HA}$ was subcloned from a previously reported construct (31). BORF2-3xFlag has also been reported (32).

\section{Episomal base editing experiments}

Semi-confluent 293T cells in a six-well plate format were transfected with 200 ng gRNA, 400 ng reporter, 600 ng Cas9n-UGI-NLS, and either $600 \mathrm{ng}$ pcDNA3.1-3xHA, 300 ng pcDNA3.1-3xHA, and 300 ng A3B-3xHA or $600 \mathrm{ng}$ A3B-3xHA (25 min at RT with 3:1 ratio of TransIT LT1 [Mirus] and $250 \mu \mathrm{l}$ of serum-free RPMI 1640 [Hyclone]). The cells were harvested after $72 \mathrm{~h}$ of incubation for editing quantification by flow cytometry.

\section{Chromosomal base editing experiments}

Semi-confluent 10-cm plates of 293T cells were transfected with $8 \mu \mathrm{g}$ of an HIV-1 Gag-Pol packaging plasmid, $1.5 \mu \mathrm{g}$ of a VSV-G expression plasmid, and $3 \mu \mathrm{g}$ of pLenti-CMV-mCherry-T2A-eGFP Leuzoz-IRESHygro. Viruses were harvested $48 \mathrm{~h}$ post-transfection and used to transduce target cells. $48 \mathrm{~h}$ post-transduction, the cells were selected using $250 \mu \mathrm{g} / \mathrm{ml}$ hygromycin. Transduced, mCherry-positive cells were transfected with 600 ng Cas9n-UGI editor, 200 ng of Leu202 or NS gRNA, and either 600 ng pcDNA3.1-3xHA, 300 ng pcDNA3.1-3xHA, and 300 ng A3B-3xHA or 600 ng A3B-3xHA. The cells were harvested 72 $\mathrm{h}$ post-transfection, and editing was quantified by flow cytometry (fraction of eGFP and mCherry double-positive cells in the total mCherry-positive population). For EMX1 and FANCF targets, transduced, mCherry-positive cells were transfected with 600 ng Cas9nUGl editor, 200 ng of Leu202 gRNA, and 200 ng of EMX1 or FANCF gRNA or just $200 \mathrm{ng}$ of the NS gRNA and $600 \mathrm{ng}$ A3B-3xHA. The cells were harvested $72 \mathrm{~h}$ post-transfection, and editing was quantified by flow cytometry (fraction of eGFP and mCherry double-positive cells in the total mCherry-positive population). FACS was used to collect both the

panel (B). (E) Quantification of eGFP-positive 293T cells (eGFP Leu202 edited with co-delivery of EMX1 gRNA) post-editing and pre-enrichment by FACS for the indicated editing reactions ( $n=3$ technical replicate experiments, average \pm SD). (E, F) Sequence logos summarizing MiSeq data of EMX1 from the same reactions as panel (E). The consensus sequence matches the single-stranded DNA region displaced by gRNA annealing with the target cytosine. Red coloring highlights base substitution mutations that occurred in $>5 \%$ of the MiSeq reads for each reaction (numbers are nucleobase distances $5^{\prime}$ or $3^{\prime}$ of the target “ $C^{\prime \prime}$ ). ( $\left.\mathbf{F}, \mathbf{G}\right)$ Quantification of single nucleobase substitution mutations from the MagnEdit reaction shown in panel (F). (F, H) Quantification of single nucleobase substitution mutations from the CBE reaction shown in panel (F). 
mCherry-positive and the eGFP and mCherry double-positive cells for genomic DNA isolation and MiSeq analysis.

\section{MiSeq}

eGFP target sequences were amplified using Phusion high-fidelity DNA polymerase (New England Biolabs) and previously reported primers (13). To add diversity to the sequence library, zero, one, or two extra cytosine bases were added to the $5^{\prime}$ end of the forward and reverse primers for each amplicon. Barcodes were added to generate full-length Illumina amplicons. Samples were analyzed using Illumina MiSeq $2 \times 150$-nucleotide paired-end reads (University of Minnesota Genomics Center). Reads were paired using FLASh (43). Data processing was performed using a locally installed FASTX-Toolkit. Fastx-clipper was used to trim the $3^{\prime}$ constant adapter region from sequences, and a stand-alone script was used to trim $5^{\prime}$ constant regions. Trimmed sequences were then filtered for high-quality reads using the Fastx-quality filter. Sequences with a Phred quality score less than 30 (99.9\% base calling accuracy) at any position were eliminated. Preprocessed sequences were then further analyzed using the FASTAptamer toolkit (44). FASTAptamer-Count was used to determine the number of times each sequence was sampled from the population. Each sequence was then ranked and sorted based on overall abundance, normalized to the total number of reads in each population, and directed into FASTAptamer-Enrich. FASTAptamerEnrich calculates the fold enrichment ratios from a starting population to a selected population by using the normalized reads-per-million (RPM) values for each sequence. For eGFP Leu202 reporter and edited sequence comparison, sequences over 5 RPM were included and only on-target sequences were used for Fig 3B. For EMX1 and FANCF analysis, all sequences over 5 RPM were included (Fig 4).

\section{Immunoblots}

$1 \times 10^{6}$ cells were lysed directly into $2.5 \times$ Laemmli sample buffer, separated by $4-20 \%$ SDS-PAGE, and transferred to PVDF-FL membranes (Millipore). The membranes were blocked in $5 \%$ milk in PBS and incubated with a primary antibody diluted in $5 \%$ milk in PBS supplemented with $0.1 \%$ Tween 20 . Secondary antibodies were diluted in $5 \%$ milk in PBS supplemented with $0.1 \%$ Tween 20 and $0.01 \%$ SDS. The membranes were imaged with an LI-COR Odyssey instrument. Primary antibodies used in these experiments were rabbit anti-Cas9 (ab189380; Abcam), mouse anti-tubulin (T5168; Sigma-Aldrich), rabbit anti-HA (3724S; Cell Signaling), and mouse anti-Flag (F1804; Sigma-Aldrich). Secondary antibodies used were goat anti-rabbit IRdye 800CW (82708365; LI-COR) and goat anti-mouse IRdye 680LT (925-68020; LI-COR).

\section{Data access}

The sequencing data generated from both CBE and MagnEdit editing studies are available at ArrayExpress (E-MTAB-8742).

\section{Supplementary Information}

Supplementary Information is available at https://doi.org/10.26508/lsa. 201900606.

\section{Acknowledgements}

We thank David Liu for providing BE3 and University of Minnesota Center for Genome Engineering colleagues for helpful comments. These studies were supported in part by a grant from the National Cancer Institute (P01CA234228 to RS Harris). Salary support for JL McCann was provided in part by the National Science Foundation Graduate Research Fellowship (Grant Number 00039202) and for DJ Salamango from the University of Minnesota Craniofacial Research Training (MinnCResT) program (T90-DE022732) and National Institute of Allergy and Infectious Disease K99/R00 (K99-Al147811). RS Harris is the Margaret Harvey Schering Land Grant Chair for Cancer Research, a Distinguished University McKnight Professor and an Investigator of the Howard Hughes Medical Institute.

\section{Author Contributions}

JL McCann: conceptualization, formal analysis, validation, investigation, methodology, and writing-original draft, review, and editing.

D) Salamango: conceptualization, formal analysis, funding acquisition, validation, investigation, methodology, and writing-review and editing.

EK Law: investigation, methodology, and writing-review and editing.

WL Brown: investigation, methodology, project administration, and writing-review and editing.

RS Harris: conceptualization, formal analysis, supervision, funding acquisition, investigation, project administration, and writing-original draft, review, and editing.

\section{Conflict of Interest Statement}

RS Harris is a co-founder, shareholder, and consultant for ApoGen Biotechnologies Inc. The other authors have no conflicts of interest to declare.

\section{References}

1. Komor AC, Kim YB, Packer MS, Zuris JA, Liu DR (2016) Programmable editing of a target base in genomic DNA without double-stranded DNA cleavage. Nature 533: 420-424. doi:10.1038/nature17946

2. Gaudelli NM, Komor AC, Rees HA, Packer MS, Badran AH, Bryson DI, Liu DR (2017) Programmable base editing of $A \cdot T$ to $G \cdot C$ in genomic DNA without DNA cleavage. Nature 551: 464-471. doi:10.1038/nature24644

3. Grünewald J, Zhou R, Garcia SP, Iyer S, Lareau CA, Aryee MJ, Joung JK (2019) Transcriptome-wide off-target RNA editing induced by CRISPR-guided DNA base editors. Nature 569: 433-437. doi:10.1038/s41586-019-1161-z

4. Zhou C, Sun Y, Yan R, Liu Y, Zuo E, Gu C, Han L, Wei Y, Hu X, Zeng R, et al (2019) Off-target RNA mutation induced by DNA base editing and its elimination by mutagenesis. Nature 571: 275-278. doi:10.1038/s41586019-1314-0

5. Kim D, Lim K, Kim ST, Yoon SH, Kim K, Ryu SM, Kim JS (2017) Genome-wide target specificities of CRISPR RNA-guided programmable deaminases. Nat Biotechnol 35: 475-480. doi:10.1038/nbt.3852

6. Gehrke JM, Cervantes O, Clement MK, Wu Y, Zeng J, Bauer DE, Pinello L, Joung JK (2018) An APOBEC3A-Cas9 base editor with minimized bystander and off-target activities. Nat Biotechnol 36: 977-982. doi:10.1038/nbt.4199

7. Zuo E, Sun Y, Wei W, Yuan T, Ying W, Sun H, Yuan L, Steinmetz LM, Li Y, Yang H (2019) Cytosine base editor generates substantial off-target 
single-nucleotide variants in mouse embryos. Science 364: 289-292 doi:10.1126/science.aav9973

8. Jin S, Zong Y, Gao Q, Zhu Z, Wang Y, Qin P, Liang C, Wang D, Qiu JL, Zhang F, et al (2019) Cytosine, but not adenine, base editors induce genome-wide off-target mutations in rice. Science 364: 292-295. doi:10.1126/ science.aaw7166

9. Coelho MA, Li S, Pane LS, Firth M, Ciotta G, Wrigley JD, Cuomo ME, Maresca M, Taylor BJM (2018) BE-FLARE: A fluorescent reporter of base editing activity reveals editing characteristics of $A P O B E C 3 A$ and $A P O B E C 3 B$. BMC Biol 16: 150. doi:10.1186/s12915-018-0617-1

10. Kim YB, Komor AC, Levy JM, Packer MS, Zhao KT, Liu DR (2017) Increasing the genome-targeting scope and precision of base editing with engineered Cas9-cytidine deaminase fusions. Nat Biotechnol 35: 371-376. doi:10.1038/nbt.3803

11. Koblan LW, Doman JL, Wilson C, Levy JM, Tay T, Newby GA, Maianti JP, Raguram A, Liu DR (2018) Improving cytidine and adenine base editors by expression optimization and ancestral reconstruction. Nat Biotechnol 36: 843-846. doi:10.1038/nbt.4172

12. St Martin A, Salamango D, Serebrenik A, Shaban N, Brown WL, Donati F, Munagala U, Conticello SG, Harris RS (2018) A fluorescent reporter for quantification and enrichment of DNA editing by APOBEC-Cas9 or cleavage by Cas9 in living cells. Nucleic Acids Res 46: e84. doi:10.1093/ nar/gky332

13. St Martin AR, Salamango DJ, Serebrenik AA, Shaban NM, Brown WL, Harris RS (2019) A panel of eGFP reporters for single base editing by APOBECCas9 editosome complexes. Sci Rep 9: 497. doi:10.1038/s41598-01836739-9

14. Zong Y, Song Q, Li C, Jin S, Zhang D, Wang Y, Qiu JL, Gao C (2018) Efficient C-to-T base editing in plants using a fusion of nCas9 and human APOBEC3A. Nat Biotechnol 36: 950-953. doi:10.1038/nbt.4261

15. Wang X, Li J, Wang Y, Yang B, Wei J, Wu J, Wang R, Huang X, Chen J, Yang L (2018) Efficient base editing in methylated regions with a human APOBEC3A-Cas9 fusion. Nat Biotechnol 36: 946-949. doi:10.1038/nbt.4198

16. Komor AC, Zhao KT, Packer MS, Gaudelli NM, Waterbury AL, Koblan LW, Kim YB, Badran AH, Liu DR (2017) Improved base excision repair inhibition and bacteriophage Mu Gam protein yields C:G-to-T:A base editors with higher efficiency and product purity. Sci Adv 3: eaao4774. doi:10.1126/sciadv.aao4774

17. Ma Y, Zhang J, Yin W, Zhang Z, Song Y, Chang X (2016) Targeted AIDmediated mutagenesis (TAM) enables efficient genomic diversification in mammalian cells. Nat Methods 13: 1029-1035. doi:10.1038/nmeth.4027

18. Hess GT, Fresard L, Han K, Lee CH, Li A, Cimprich KA, Montgomery SB, Bassik MC (2016) Directed evolution using dCas9-targeted somatic hypermutation in mammalian cells. Nat Methods 13: 1036-1042. doi:10.1038/nmeth.4038

19. Hu JH, Miller SM, Geurts MH, Tang W, Chen L, Sun N, Zeina CM, Gao X, Rees HA, Lin Z, et al (2018) Evolved Cas9 variants with broad PAM compatibility and high DNA specificity. Nature 556: 57-63. doi:10.1038/nature26155

20. Thuronyi BW, Koblan LW, Levy JM, Yeh WH, Zheng C, Newby GA, Wilson C, Bhaumik M, Shubina-Oleinik O, Holt JR, et al (2019) Continuous evolution of base editors with expanded target compatibility and improved activity. Nat Biotechnol 37: 1070-1079. doi:10.1038/s41587-019-0193-0

21. Huang TP, Zhao KT, Miller SM, Gaudelli NM, Oakes BL, Fellmann C, Savage DF, Liu DR (2019) Author Correction: Circularly permuted and PAMmodified Cas9 variants broaden the targeting scope of base editors. Nat Biotechnol 37: 820. doi:10.1038/s41587-019-0168-1

22. Rees HA, Komor AC, Yeh WH, Caetano-Lopes J, Warman M, Edge ASB, Liu DR (2017) Improving the DNA specificity and applicability of base editing through protein engineering and protein delivery. Nat Commun 8: 15790. doi:10.1038/ncomms15790

23. Endo M, Mikami M, Endo A, Kaya H, Itoh T, Nishimasu H, Nureki O, Toki S (2019) Genome editing in plants by engineered CRISPR-Cas9 recognizing NG PAM. Nat Plants 5: 14-17. doi:10.1038/s41477-018-0321-8
24. Li X, Wang Y, Liu Y, Yang B, Wang X, Wei J, Lu Z, Zhang Y, Wu J, Huang X, et al (2018) Base editing with a Cpf1-cytidine deaminase fusion. Nat Biotechnol 36: 324-327. doi:10.1038/nbt.4102

25. Kleinstiver BP, Sousa AA, Walton RT, Tak YE, Hsu JY, Clement K, Welch MM Horng JE, Malagon-Lopez J, Scarfó I, et al (2019) Engineered CRISPRCas12a variants with increased activities and improved targeting ranges for gene, epigenetic and base editing. Nat Biotechnol 37: 276-282. doi:10.1038/s41587-018-0011-0

26. Land AM, Wang J, Law EK, Aberle R, Kirmaier A, Krupp A, Johnson WE, Harris RS (2015) Degradation of the cancer genomic DNA deaminase APOBEC3B by SIV Vif. Oncotarget 6: 39969-39979. doi:10.18632/ oncotarget.5483

27. Zhang W, Zhang X, Tian C, Wang T, Sarkis PT, Fang Y, Zheng S, Yu XF, Xu R (2008) Cytidine deaminase APOBEC3B interacts with heterogeneous nuclear ribonucleoprotein $\mathrm{K}$ and suppresses hepatitis $\mathrm{B}$ virus expression. Cell Microbiol 10: 112-121. doi:10.1111/j.14625822.2007.01020.x

28. McCann JL, Klein MM, Leland EM, Law EK, Brown WL, Salamango DJ, Harris RS (2019) The DNA deaminase APOBEC3B interacts with the cell-cycle protein CDK4 and disrupts CDK4-mediated nuclear import of Cyclin D1. Biol Chem 294: 12099-12111. doi:10.1074/jbc.RA119.008443

29. Lackey L, Demorest ZL, Land AM, Hultquist JF, Brown WL, Harris RS (2012) APOBEC3B and AID have similar nuclear import mechanisms. J Mol Biol 419: 301-314. doi:10.1016/j.jmb.2012.03.011

30. Lackey L, Law EK, Brown WL, Harris RS (2013) Subcellular localization of the APOBEC3 proteins during mitosis and implications for genomic DNA deamination. Cell Cycle 12: 762-772. doi:10.4161/cc.23713

31. Salamango DJ, McCann JL, Demir O, Brown WL, Amaro RE, Harris RS (2018) APOBEC3B nuclear localization requires two distinct $\mathrm{N}$-terminal domain surfaces. J Mol Biol 430: 2695-2708. doi:10.1016/j.jmb.2018.04.044

32. Cheng AZ, Yockteng-Melgar J, Jarvis MC, Malik-Soni N, Borozan I, Carpenter MA, McCann JL, Ebrahimi D, Shaban NM, Marcon E, et al (2019) Epstein-Barr virus BORF2 inhibits cellular APOBEC3B to preserve viral genome integrity. Nat Microbiol 4: 78-88. doi:10.1038/s41564-0180284-6

33. Shi K, Carpenter MA, Banerjee S, Shaban NM, Kurahashi K, Salamango DJ, McCann JL, Starrett GJ, Duffy JV, Demir O, et al (2017) Structural basis for targeted DNA cytosine deamination and mutagenesis by APOBEC3A and APOBEC3B. Nat Struct Mol Biol 24:131-139. doi:10.1038/ nsmb.3344

34. Bennett RP, Diner E, Sowden MP, Lees JA, Wedekind JE, Smith HC (2006) APOBEC-1 and AID are nucleo-cytoplasmic trafficking proteins but APOBEC3G cannot traffic. Biochem Biophys Res Commun 350: 214-219. doi:10.1016/j.bbrc.2006.09.032

35. Patenaude AM, Orthwein A, Hu Y, Campo VA, Kavli B, Buschiazzo A, Di Noia JM (2009) Active nuclear import and cytoplasmic retention of activation-induced deaminase. Nat Struct Mol Biol 16: 517-527. doi:10.1038/nsmb.1598

36. Shi K, Demir O, Carpenter MA, Wagner J, Kurahashi K, Harris RS, Amaro RE, Aihara H (2017) Conformational switch regulates the DNA cytosine deaminase activity of human APOBEC3B. Sci Rep 7: 17415. doi:10.1038/ s41598-017-17694-3

37. Wagner JR, Demir Ö, Carpenter MA, Aihara H, Harki DA, Harris RS, Amaro RE (2019) Determinants of oligonucleotide selectivity of APOBEC3B. Chem Inf Model 59: 2264-2273. doi:10.1021/acs.jcim.8b00427

38. Huang YH, Su J, Lei Y, Brunetti L, Gundry MC, Zhang X, Jeong M, Li W, Goodell MA (2017) DNA epigenome editing using CRISPR-Cas SunTagdirected DNMT3A. Genome Biol 18: 176. doi:10.1186/s13059-017-1306-Z

39. Tanenbaum ME, Gilbert LA, Qi LS, Weissman JS, Vale RD (2014) A protein-tagging system for signal amplification in gene expression and fluorescence imaging. Cell 159: 635-646. doi:10.1016/ j.cell.2014.09.039 
40. Yoon JH, Srikantan S, Gorospe M (2012) MS2-TRAP (MS2-tagged RNA affinity purification): Tagging RNA to identify associated miRNAs. Methods 58: 81-87. doi:10.1016/j.ymeth.2012.07.004

41. Wang J, Shaban NM, Land AM, Brown WL, Harris RS (2018) Simian immunodeficiency virus Vif and human APOBEC3B interactions resemble those between HIV-1 Vif and human APOBEC3G. J Virol 92 e00447. doi:10.1128/JVI.00447-18

42. Carpenter MA, Law EK, Serebrenik A, Brown WL, Harris RS (2019) A lentivirus-based system for Cas9/gRNA expression and subsequent removal by Cre-mediated recombination. Methods 156: 79-84. doi:10.1016/j.ymeth.2018.12.006
43. Magoc T, Salzberg SL (2011) FLASH: Fast length adjustment of short reads to improve genome assemblies. Bioinformatics 27: 2957-2963. doi:10.1093/ bioinformatics/btr507

44. Alam KK, Chang JL, Burke DH (2015) FASTAptamer: A bioinformatic toolkit for high-throughput sequence analysis of combinatorial selections. Mol Ther Nucleic Acids 4: e230. doi:10.1038/mtna.2015.4

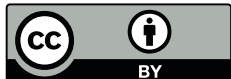

License: This article is available under a Creative Commons License (Attribution 4.0 International, as described at https://creativecommons.org/ licenses/by/4.0/). 